\title{
Detection of human papillomavirus and expression of osteopontin in cervical cancer specimens
}

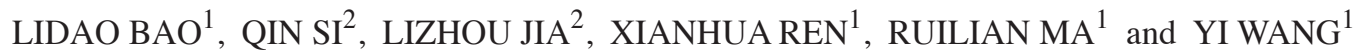 \\ ${ }^{1}$ Department of Pharmacy, Affiliated Hospital of Inner Mongolia Medical University, Hohhot, Inner Mongolia 010059, \\ P.R. China; ${ }^{2}$ Medical Oncology, Dana-Farber Cancer Institute, Harvard Medical School, Boston, MA 02120, USA
}

Received January 14, 2014; Accepted August 14, 2014

DOI: $10.3892 / \mathrm{mmr} .2014 .2647$

\begin{abstract}
To understand human papillomavirus (HPV) and expression of osteopontin (OPN) in cervical diseased tissues, HPV infection was detected in paraffin-embedded specimens of cervical lesions from 90 patients with cervical cancer. Three polymerase chain reaction (PCR) techniques were used to determine the detectable rate of HPV infection. Expression of HPV OPN protein was detected using immunohistochemical methods. When a pairwise comparison was made among the three PCR methods, $\chi^{2}$ analysis indicated $\mathrm{P}>0.05$ for detection through the methods of MY09/11 and $\mathrm{GP}^{+} / 6^{+}$, and $\mathrm{P}>0.05$ through the methods of MY09/11 and Nested-PCR. This indicated that there was no statistically significant difference in the HPV infection detection sensitivity of these methods. However, $\chi^{2}$ comparison of the methods of $\mathrm{GP}^{+} / 6^{+}$and Nested-PCR indicated $\mathrm{P}<0.05$, which demonstrated that there was a statistically significant difference. The rate of positive HPV DNA measured with Nested-PCR was significantly higher than that measured using the GP5 ${ }^{+} / 6^{+}$PCR method. The HPV OPN protein is expressed in cervical cancer, and the HPV OPN polypeptide antibody has broad spectrum reaction capacity and significant multivalence for HPV infection. Immunohistochemical detection was performed on tissue specimens using the purified rabbit HPV OPN polypeptide antibody. Sixty-one cases exhibited a positive result and 29 a negative result. The total rate of positive detection was $67.78 \%$. HPV OPN may therefore serve as a candidate target for tumor treatment, including targeted therapies and vaccine development.
\end{abstract}

Correspondence to: Professor Yi Wang, Department of Pharmacy, Affiliated Hospital of Inner Mongolia Medical University, 1 Tongdaobei Street, Hohhot, Inner Mongolia 010059, P.R. China E-mail: wang_yi237@163.com

Key words: cervical cancer, human papillomavirus typing, osteopontin, expression

\section{Introduction}

Human papillomavirus (HPV) is a small double-stranded circular DNA virus infecting the squamous epithelial cells of the skin and mucosa. It is able to immortalize normal cells and infect human skin and epithelia, including the genital and respiratory tracts. HPV is associated with numerous benign and malignant tumors $(1,2)$. Cervical cancer has become the second largest malignant tumor to threaten female life, following breast cancer. The morbidity and mortality of cervical cancer in China account for approximately one-third of those worldwide. The overall morbidity rate is $0.27 \%$ (3). Based on epidemiological and biological studies, it is clear that HPV infection is a required condition for pre-cervical cancer lesions and the occurrence of invasive cervical cancer (4). High-risk HPV (HrHPV), including HPV31, 33 and 35, and particularly HPV16 and 18 , are closely associated with the occurrence of cervical cancer and cervical intraepithelial neoplasia (CIN). HrHPV may be responsible for $>95 \%$ of cervical cancer cases $(5,6)$.

Osteopontin (OPN) is a phosphorylated glycoprotein, which bridges cell and matrix inorganic substances and is closely associated with the occurrence, progression and metastasis of malignant tumors (7). Recently, certain studies have indicated that OPN is expressed in cervical cancer $(8,9)$. OPN is associated with positive expression rate, tumor staging, lymphatic metastasis and distant metastasis in human cervical cancer. In normal cervical mucosa, OPN is similarly expressed in CIN Grade I and Grade II groups. However, OPN expression is increased in CIN Grade III patients, particularly in infiltrating tumor tissues. Hence, OPN may promote tumor metastasis (10).

Therefore, the aims of the present study were to explore the expression of HPV OPN in diseased cervical tissues to determine whether HPV OPN may be able to serve as a target protein in tumor treatment. Furthermore, the present study aimed to determine whether the HPV OPN C-terminal common sequence polypeptide antibody was able to accurately detect HPV OPN. These results may provide theoretical and experimental support for HPV vaccine development and potential targeted therapies for cervical tumors.

\section{Materials and methods}

Subjects. Ninety diseased cervical tissue specimens were collected from the Pathology Department of People's Hospital 
Affiliated to Inner Mongolia Medical University (Hohhot, China) from June 2012 to May 2013. The present study was approved by the Ethics Committee of the People's Hospital Affiliated to Inner Mongolia Medical University. Consent was obtained from all enrolled patients prior to examination and treatment according to the Declaration of Helsinki and relevant laws in China. All treatments were performed based on the patients' best interests.

Pathological and cytological classification of cervical lesions was performed for all specimens. High-grade squamous intraepithelial lesion (HSIL) included CIN Grade 2 (CIN2) and CIN Grade 3 (CIN3) and carcinomas in situ were classified as CIN3; low-grade squamous intraepithelial lesion (LSIL) was equivalent to CIN Grade 1 (CIN1) (11). The types and number of cases of cervical cancer detected amongst the 90 patients were as follows: Cervical squamous cell carcinoma, 38 cases; adenocarcinoma of the cervix, 23 cases; HSIL (CIN2, CIN3 and carcinoma in situ), 18 cases; LSIL (CIN1), 11 cases.

The tissue samples were paraffin-embedded, sectioned into 10- $\mu \mathrm{m}$ sections and placed into Eppendorf (EP) tubes at a density of five sections per tube. Each tube was sealed using sealing film prior to HPV DNA level detection. In addition, each paraffin-embedded specimen was serially sectioned and prepared for immunohistochemical analysis. All specimens were stored in a refrigerator at $4^{\circ} \mathrm{C}$.

Reagents and instruments. Tris was purchased from Fermentas (Thermo Fisher Scientific, Pittsburgh, PA, USA); proteinase K was purchased from Biowish Biotechnology Co., Ltd. (Hangzhou, China); agarose was purchased from Beijing Bole Bioscience Development Co., Ltd. (Beijing, China); ethidium bromide was purchased from Shanghai Meiji biotechnology Co., Ltd. (Shanghai, China); DNA marker and 2XTaq Master Mix were purchased from Shanghai Boyan Biotechnology Co., Ltd. (Shanghai, China); DNA amplification reagent kits and typing detection reagent kits for HPV nucleic acid amplification were purchased from Invitrogen Life Technologies (Carlsbad, CA, USA); the PCR amplifier was purchased from Labnet International, Inc. Global (Edison, NJ, USA) and the PTC-200 Eppendorf Master ThermalCycler hybridization instrument was purchased from Eppendorf (Hamburg, Germany).

Total DNA extraction from specimens. The paraffin-embedded specimens were removed from the $1.5 \mathrm{ml} \mathrm{EP}$ tubes for marking. $250 \mu 1$ cell lysis buffer solution was added to each EP tube and the tubes were re-sealed and heated in a dry thermostat at $100^{\circ} \mathrm{C}$ for $10 \mathrm{~min}$. Once the paraffin had completely melted, the tubes were centrifuged at $12,927 \mathrm{x}$ g for $10 \mathrm{~min}$, removed and placed in an environment at $-20^{\circ} \mathrm{C}$ to allow for sufficient rigidification of the upper wax sheets. The wax sheets were removed and $2 \mu \mathrm{l}$ proteinase $\mathrm{K}(20 \mathrm{mg} / \mathrm{ml})$ was added to the sufficiently dewaxed specimens which were subsequently placed in the dry thermostat at $48^{\circ} \mathrm{C}$ for $72 \mathrm{~h}$ until the solutions became clear. The specimens were then heated in the dry thermostat at $100^{\circ} \mathrm{C}$ for $10 \mathrm{~min}$ to inactivate the protease and centrifuged at $12,927 \mathrm{x} \mathrm{g}$ for $10 \mathrm{~min}$. The supernatants were transferred to $0.5 \mathrm{ml} \mathrm{EP}$ tubes and stored at $4^{\circ} \mathrm{C}$ in preparation for PCR and HPV gene amplification hybridization typing detection or stored at $-20^{\circ} \mathrm{C}$ for later use.
Primer synthesis. Primers were synthesized by Shanghai Yanjing Biotechnology Co., Ltd (Shanghai, China). Referring to the complete sequence of the HPV gene, the primer 5.0 primer design software (Premier Biosoft, Palo Alto, CA, USA) and DNAman gene analysis software 5.2.9 Demo version (Lynnon LLC, San Ramon, CA, USA) were used in order to detect the primer location and to screen the appropriate primers (12). MY09/11 upstream primer, 5'-CGTCCTGGATATGCTGTATGC-3' and downstream, 5'-GAACCAGGGCATATAAATGG-3', 384 bp. GP5 ${ }^{+} / 6^{+}$ upstream, 5'-TYACGTTACTTAGATACTGT-3' and downstream, 5' GATCATAAACTATAAAGTAAC-3', 152 bp (mixed base: $\mathrm{M}=\mathrm{A}+\mathrm{C}, \mathrm{R}=\mathrm{A}+\mathrm{G}, \mathrm{W}=\mathrm{A}+\mathrm{T}, \mathrm{Y}=\mathrm{C}+\mathrm{T}$ ).

The internal reference $\beta$-globin gene infected with HPV in human host cells was selected as a control and its primers were as follows: Upstream, 5'-ACTAAACTGCAGTTCACGTC-3' and downstream, 5'-ATCCACTGTACATCACTCCC-3'.

Experimental grouping. The present study comprised three experimental groups for PCR analysis; the experimental group, total DNA extracted from the clinical cervical paraffin specimens; the positive control group, total DNA from SiHa cells (HPV16-positive cervical squamous epithelial cell line and viral genome integration) and the negative control group, total DNA from HeCaT cells (normal human immortalized cutin cells). SiHa and $\mathrm{HeCaT}$ cell lines were purchased from Shanghai Enzyme-linked Biotechnology Co., Ltd. (Shanghai, China).

Polymerase chain reaction (PCR) amplification of $\beta$-globin, OPN conserved sequence general-type primer MY09/11 and OPN conserved sequence general-type primer $G P 5^{+} / 6^{+}$. Nested PCR was performed by first amplifying with MY09/11,GP5+/6+ primers, followed by a second amplification with the internal standard of human host cells infected by HPV, i.e. the $\beta$-globin gene. DNA was extracted using a Total DNA extraction kit (Qiagen, Hilden, Germany) The PCR reaction mixture contained: $12.5 \mu \mathrm{l}$ 2XTaq Master Mix, $0.5 \mu \mathrm{l}$ upstream and downstream primers of HPV $\beta$-globin gene, HPV OPN general-type MY09/11 or HPV OPN general-type $\mathrm{GP}^{+} / 6^{+}(10 \mu \mathrm{mol} / \mathrm{l})$ and $2 \mu 1$ template. Deionized water was added to make the solution up to $25 \mu 1$, which was then mixed and centrifuged slightly.

PCR reaction conditions: Initial denaturation at $94^{\circ} \mathrm{C}$ for $5 \mathrm{~min}$; denaturation at $94^{\circ} \mathrm{C}$ for $45 \mathrm{sec}$; annealing at $55^{\circ} \mathrm{C}$ for $45 \mathrm{sec}$ and extension at $72^{\circ} \mathrm{C}$ for $90 \mathrm{sec}$ for 30 cycles in total. Final extension was at $72^{\circ} \mathrm{C}$ for $5 \mathrm{~min}$.

Detection of PCR products: $5 \mu \mathrm{l}$ PCR reaction products and $1 \mu \mathrm{l} 6 \mathrm{X}$ bromophenol blue loading buffer were mixed, electrophoresed with $2 \%$ agarose gel at $80-90 \mathrm{~V}$ for $1 \mathrm{~h}$, scanned and photographed using a Gel.Doc2000 gel imager (Bio-Rad, Hercules, CA, USA).

Immunohistochemical experimental method for HPV OPN polypeptide antibody. The prepared pathological sections were placed in an oven at $70^{\circ} \mathrm{C}$ for $15 \mathrm{~min}$. Subsequently, the section rack with the sections mounted on it was placed in a dewaxing jar with dimethylbenzene solution (Ji'nan Huifengda Chemical Co.,Ltd., Ji'nan, China) and dewaxed twice for 15 min each time. The dimethylbenzene solution was removed, 
sections were placed in clean distilled water and soaked for several min, placed in the ready-to-use IHC antigen retrieval solution, 100x (Beijing ComWin Biotech Co., Ltd., Beijing, China), heated to $100^{\circ} \mathrm{C}$ for $1 \mathrm{~min}$, removed and stored at room temperature for natural cooling. Sections were soaked in phosphate buffered saline (PBS) for $10 \mathrm{~min}$, subsequently the glass slides around the tissue were wiped dry and soaked for $30 \mathrm{~min}$ following addition of $3 \% \mathrm{H}_{2} \mathrm{O}_{2}$ (Ji'nan Huifengda Chemical Co.,Ltd.). Sections were agitated and washed with phosphate buffer three times for three minutes each time and blocked in confining liquid at room temperature for $1 \mathrm{~h}$. All antibodies were obtained from ChinaPeptides Co., Ltd., (Shanghai, China). Rabbit HPV OPN polyclonal antibody, anti-mouse HPV OPN polyclonal antibody (primary antibody; 1:200) was added drop-wise to the sectioned tissue and incubated at room temperature for $1 \mathrm{~h}$ prior to being washed with PBS three times for five minutes each time. Anti-rabbit or anti-mouse immunoglobulin $\mathrm{G}$ biotinylated secondary antibody was subsequently added to each sectioned tissue and incubated for 10 min prior to being rinsed with PBS three times for three minutes each. Streptavidin horseradish peroxidase marker was added to cover each sectioned tissue, incubated for $10 \mathrm{~min}$ then rinsed with PBS three times for three minutes each. The appropriate amount of ready-to-use diaminobenzidine color development solution (Beijing Solarbio Co., Ltd., Beijing, China) was added to the sectioned tissue. Sections were developed away from sunlight at room temperature and the color development was observed under a BK-DM320/500 digital biological microscope, (Leica, Germany). Sections were washed with double distilled water to terminate the reaction and tissues were counterstained with hematoxylin for $5 \mathrm{~min}$ and washed with tap water, then differentiated with hydrochloric acid alcohol and washed with tap water. Finally, the sectioned tissues were dehydrated, clarified, mounted and dried at room temperature. The experimental results were observed and recorded under a microscope (13).

Statistical analysis. All data are expressed as the mean \pm the standard error of the mean. The data obtained were subjected to statistical analysis using one-way analysis of variance followed by Dunnett's post test for comparison between control and test groups using SPSS version 12.0 software (SPSS, Inc., Chicago,IL, USA). P<0.05 was considered to indicate a statistically significant difference. For quantification of the PCR and western blot results, the gray scale pictures were quantified using Gel-Pro Analyzer. The fold changes of the mRNA and protein level were expressed relative to the readings of the control group.

\section{Results}

PCR analysis of HPV. HPV PCR amplification with $\beta$-globin as internal reference was performed for the total DNA extracted from all 90 specimens. The total DNA was electrophoresed with $2 \%$ agarose gel following amplification. A consistent band was observed at $\sim 153$ bp (Fig. 1) for all experimental specimens. The success rate of DNA extraction was therefore $100 \%$. general-type primer MY09/11. MY09/11 primer PCR amplifica-

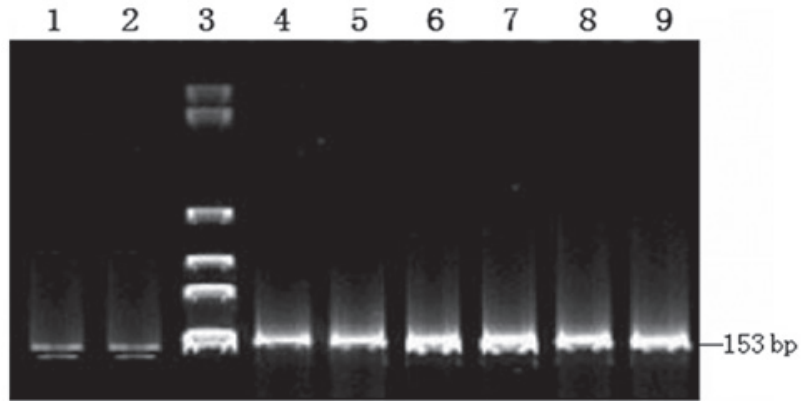

Figure 1. $\beta$-globin primer polymerase chain reaction electrophoresis. Lanes: 1, SiHa control; 2, HaCat control; 3, DNA marker; 4-9, experimental specimens.

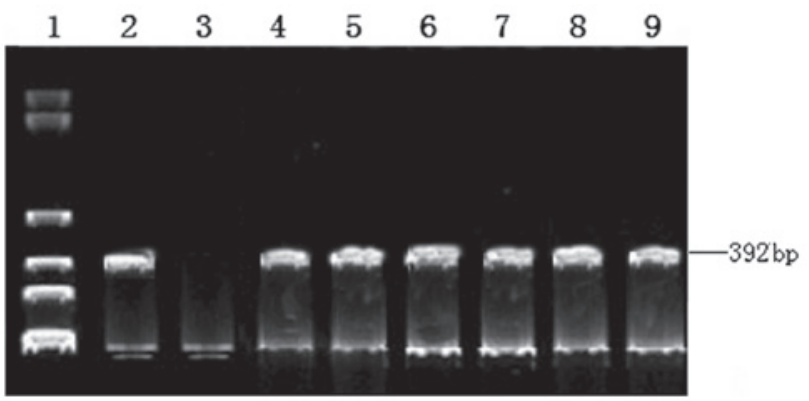

Figure 2. General-type MY09/11 primer polymerase chain reaction electrophoresis. Lanes: 1, DNA marker; 2, SiHa positive control; 3, HaCat negative control; 4-9, experimental specimens.

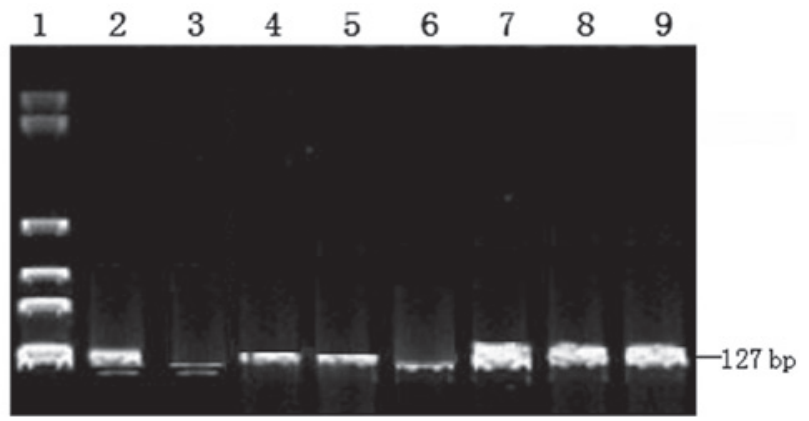

Figure 3. General-type $\mathrm{GP}^{+} / 6^{+}$primer polymerase chain reaction electrophoresis. Lanes: 1, SiHa positive control; 2, HaCat negative control; 3, is DNA marker; 4-9, experimental specimens.

tion was performed for all 90 specimens. The specimens were electrophoresed using a $2 \%$ agarose gel following amplification. A marked band at 392 bp (Fig. 2), indicating positive HPV DNA, was observed in 39 of the experimental specimens. This result indicated that there were $39 \mathrm{HPV}$-positive specimens and therefore, the detection rate of HPV infection was $43.33 \%$.

PCR amplification of HPV OPN conserved sequence using general-type primer $G P 5^{+} / 6^{+}$. GP5 $5^{+} / 6^{+}$primer amplification was performed for all specimens that were found to be HPV-negative in the MY09/11 primer PCR amplification detection. The specimens were electrophoresed using a $2 \%$ agarose gel following amplification. A band at $\sim 127$ bp (Fig. 3), indicating positive HPV DNA, was observed in 22 of the experimental specimens. 
Table I. Results of Nested-PCR examination.

\begin{tabular}{lccc}
\hline PCR Class & Number of cases tested & Number of cases of positive HPV DNA & Positive rate $(\%)$ \\
\hline MY09/11 & 90 & 39 & 43.33 \\
GP5 $^{+} / 6^{+}$ & 90 & 22 & $24.44^{\mathrm{a}}$ \\
Nested-PCR & 90 & 48 & $53.33^{\mathrm{a}}$ \\
\hline
\end{tabular}

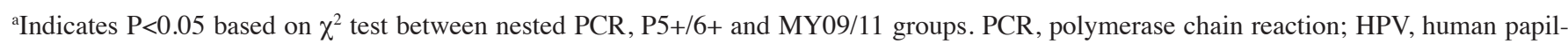
lomavirus.

Table II. Immunohistochemical results of anti-rabbit paraffin sections.

\begin{tabular}{lccc}
\hline Group & Number of cases tested & Number of positive cases & Positive rate $(\%)$ \\
\hline LSIL & 11 & 7 & 63.64 \\
HSIL & 18 & 11 & 61.11 \\
Cervical squamous cell carcinoma & 38 & 29 & 76.32 \\
Cervical adenocarcinoma & 23 & 14 & 60.87 \\
\hline
\end{tabular}

LSIL, low-grade squamous intraepithelial lesion; HSIL, high-grade squamous intraepithelial lesion.

This result indicated that there were 22 HPV-positive specimens. The detection rate of further HPV infections was $24.44 \%$.

Nested-PCR. The Nested-PCR method was performed for all specimens. This method was able to detect 48 HPV DNA-positive specimens (Table I). The total detection rate of HPV infection was therefore $53.33 \%$. The HPV detection rate obtained from amplification of two pairs of internal and external primers was significantly higher than that of amplification of either pair of primers.

Nested-PCR HPV detection rate is significantly higher than that of $G P 5^{+} / 6^{+} P C R$. When a pairwise comparison was made among the three PCR methods (Table I), $\chi^{2}$ analysis indicated $\mathrm{P}>0.05$ for detection through the methods of MY09/11 and $\mathrm{GP}^{+} / 6^{+}$; and $\mathrm{P}>0.05$ through the methods of MY09/11 and Nested-PCR. This indicated that there was no statistically significant difference in the HPV infection detection sensitivity of these methods. However, $\chi^{2}$ comparison of the methods of $\mathrm{GP}^{+} / 6^{+}$and Nested-PCR indicated $\mathrm{P}<0.05$, which demonstrated that there was a statistically significant difference. The rate of positive HPV DNA measured using the Nested-PCR method was significantly higher than that measured using the $\mathrm{GP} 5^{+} / 6^{+}$ PCR method (Table I).

Immunohistochemical detection of HPV OPN polypeptide in tissue specimens using anti-rabbit antibody. The immunohistochemical results of anti-rabbit paraffin section analysis indicated that seven of the 11 LSIL cases were HPV-positive, while 11 of the 18 HSIL cases were HPV-positive. Furthermore, 29 of the 38 cervical squamous cell carcinoma cases were HPV-positive and 14 of the 23 cervical adenocarcinoma cases were HPV-positive (Table II and Fig. 4).

The results demonstrated that overall, 61 specimens exhibited a positive reaction and 29 specimens exhibited a negative reaction. The total positive detection rate was therefore $67.78 \%$.
Immunohistochemical detection of HPV OPN polypeptide in tissue specimens using anti-mouse antibody. The immunohistochemical results of anti-mouse paraffin section analysis indicated that eight of the 11 LSIL cases were HPV-positive and 14 of the 18 HSIL cases were HPV-positive. Furthermore, 31 of the 38 cervical squamous cell carcinoma cases were HPV-positive and 15 of the 23 cervical cancer cases were HPV-positive (Table III and Fig. 5).

The results indicated that 68 specimens exhibited a positive reaction and 22 specimens exhibited a negative reaction. The total positive detection rate was therefore $75.56 \%$.

\section{Discussion}

HPV-associated cervical cancer is the second most prevalent cancer among females worldwide, the most common malignant lesion among females in developing countries and one of the major malignant tumors threatening the health of females at the present stage. Furthermore, HPV-associated cervical cancer exhibits a trend of onset in a younger age-group than other types of cancer (14). Cervical cancer poses a significant threat to the lives of females, in part because there is no accurate detection index available for predicting the occurrence and progression of the disease (15). It has been generally accepted that persistent HrHPV infection is a significant contributing factor in the development of cervical cancer; the degree of its correlation with the occurrence of cervical cancer is markedly higher than the degree of correlation between smoking and the occurrence of lung cancer (16). HPV16, 18, 31, 33, 35, 39, 45, 51, 52, 56, 58, 66, 68 and 70 have been assigned to the HrHPV group and HPV6, 11, 34, 42, 43, 44 and 74 have been assigned to the potentially carcinogenic group $(17,18)$. Pathological studies have demonstrated that there are two PCR methods (MY09/11 and $\mathrm{GP}^{+} / 6^{+}$) available for the detection and typing of HPV in cervical cancer specimens. The odds ratio of cervical cancer to HrHPV exposure 
Table III. Immunohistochemical results of anti-mouse paraffin sections.

\begin{tabular}{lccc}
\hline Group & Number of cases tested & Number of positive cases & Positive rate (\%) \\
\hline LSIL & 11 & 8 & 72.73 \\
HSIL & 18 & 14 & 77.78 \\
Cervical squamous cell carcinoma & 38 & 31 & 81.58 \\
Cervical adenocarcinoma & 23 & 15 & 65.22
\end{tabular}

LSIL, low-grade squamous intraepithelial lesion; HSIL, high-grade squamous intraepithelial lesion.
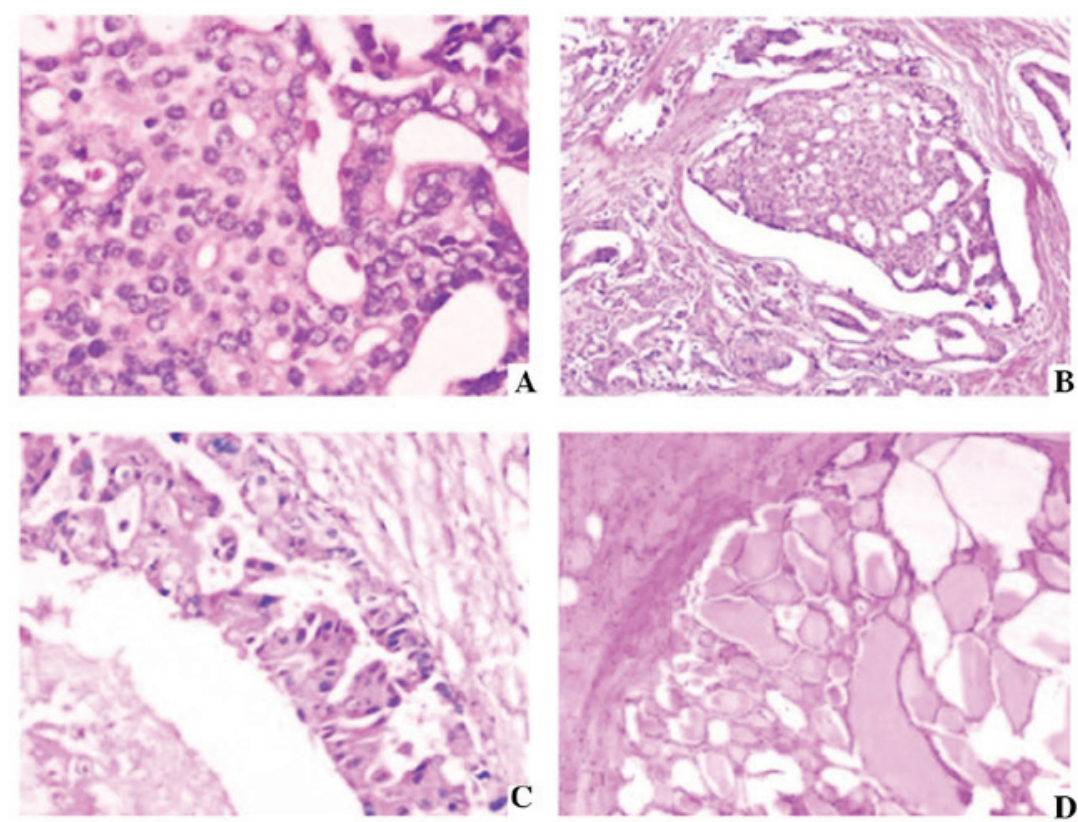

Figure 4. Anti-rabbit immunohistochemical staining for HPV OPN polypeptide. (A) Low-grade squamous intraepithelial lesion of the cervix. (B) High-grade squamous intraepithelial lesion of the cervix. (C) Cervical squamous cell carcinoma. (D) Cervical adenocarcinoma. HPV, human papillomavirus; OPN, osteopontin (magnification, x200).
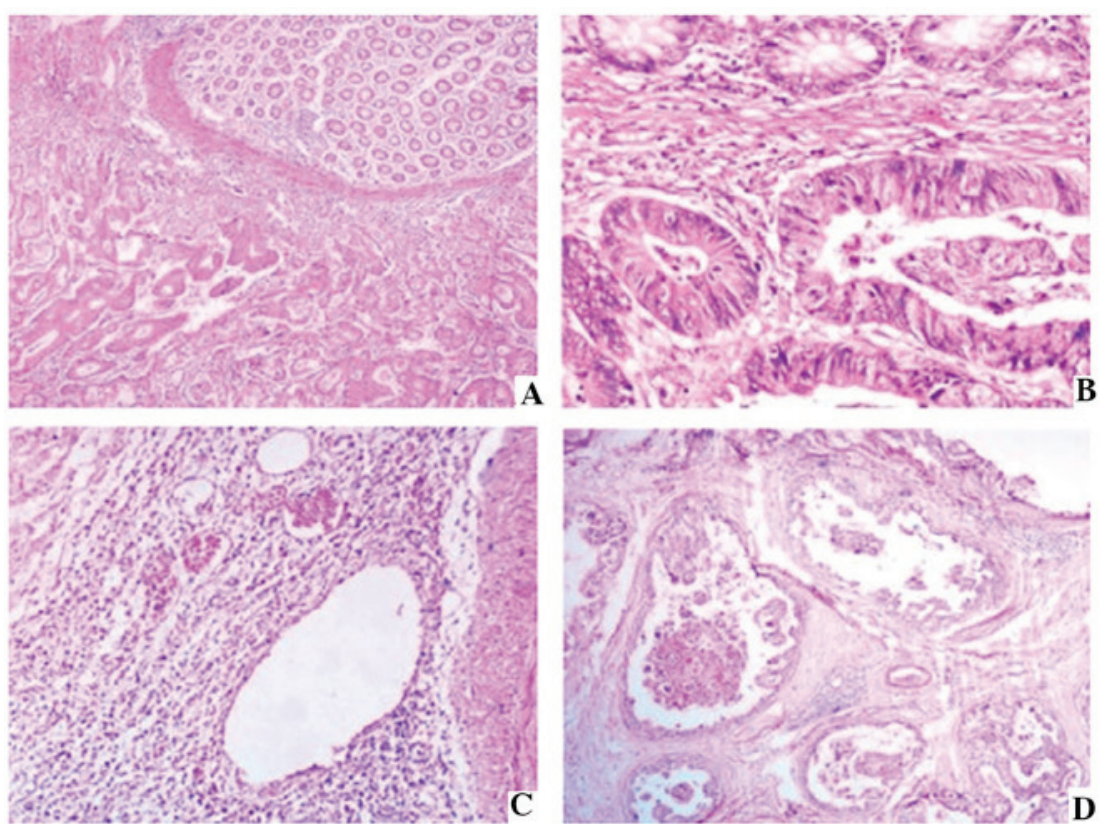

Figure 5. Anti-mouse immunohistochemical staining for HPV OPN polypeptide. (A) Low-grade squamous intraepithelial lesion of the cervix. (B) High-grade squamous intraepithelial lesion of the cervix. (C) Cervical squamous cell carcinoma. (D) Cervical adenocarcinoma. HPV, human papillomavirus; OPN, osteopontin (magnification, $\mathrm{x} 400$ ). 
obtained by the $\mathrm{GP}^{+} / 6^{+}$method is $158.2(95 \%$ confidence interval, 113.4-220.6) (19).

HPV is a spherical virus with epithelial tropism which belongs to a subgroup of the papilloma virus. HPV comprises a double-stranded closed circular DNA which specifically infects the squamous epithelial cells of human skin and mucous membranes and an icosahedral non-enveloped virus composed of core DNA and OPN. OPN is a glycoprotein and major capsid protein, which is highly conserved among HPV types and is the main group-specific antigen $(20,21)$. OPN has several important functions due to its structural characteristics and accurate antigenic characteristics, including adhesion to host cells, identification of virus receptors and assisting endocytosis and transport of viral DNA (22).

According to clinical statistics, $80 \%$ of cervical cancer tumor patients died of invasion or metastasis (23). The process of invasion and metastasis of tumor cells is complex, including a series of interactions amongst tumor cells and between tumor cells, host cells and extracellular matrixes. In particular, the involvement of multiple cytokines in cell regulation allows for metastasis and rapid growth of tumor cells $(24,25)$. OPN is one such cytokine, and functions as a secretory multi-functional phosphorylated sialoglycoprotein and a non-collagenic adhesion molecule in the extracellular matrix (26). Markman (27) suggested that expression of OPN in breast cancer was associated with clinicopathologic factors, including tumor size, histological grading and lymph node conditions. A study by Fröberg et al (28) indicated that overexpression of OPN messenger RNA in liver cancer is associated with the levels of serum alpha-fetoprotein, tumor size and clinical staging.

The outcome of a HPV-infected host is dependent on whether a rapid and intensive cellular immune response occurs at the host infection site (29). Numerous studies have indicated that the main capsid protein of HPV is the invasion site where the host-cell immune response targets HPV. OPN capsid protein, host $\mathrm{T}$ cells (CD4/CD8) and major histocompatibility complex I/II form an immune complex. This activates the immune chain reaction of the organism following host invasion by HPV and high-concentration immune antibodies kill and eliminate the HPV $(30,31)$. However, when host immunity is low or the virulence is strong, HPV is able to survive in the host cells through DNA replication, RNA transcription and protein synthesis, thus leading to persistent host infection and exacerbation of lesion extent (32). A study by Origoni et al (33) demonstrated that expression of HPV OPN tends to indicate that the organism is HPV-infected and that the HPV is at the replication stage. Detection of HPV OPN protein expression in diseased cervical tissue may be capable of monitoring the reproductive cycle of the virus and also indirectly evaluating patients' infectivity to provide theoretical guidance for the clinical application of a HPV OPN protein vaccine and potential targeted therapy for tumors (34). This study used a high-sensitivity immunoenzyme technique, and the rabbit polypeptide antibody used was regarded as the first antibody for immunohistochemical detection of HPV OPN. The results demonstrated that the cytoplasm and karyons of certain cells in the specimens exhibited a positive reaction, which indicated that these cells contained HPV OPN proteins. The cell volume was increased and the karyon was significantly enlarged in specimens positive for HPV infection.
Currently, HPV vaccine-development primarily consists of two categories: A prophylactic vaccine and a therapeutic vaccine with a main focus on HrHPV. The therapeutic vaccine may eliminate viral infection or variant cells and enhance the cell-mediated immunoreaction of infected individuals (35). Various therapeutic immunological methods are available, the most common of which involve activation of host $\mathrm{T}$ cells by tumorigenic HPV-type E6 and E7 polypeptides (36). The prophylactic vaccine is able to resist HPV infection by inducing an effective humoral immune response, mainly via the capsid protein. These components can constitute non-HPV DNA virus-like particles independently and have natural structures similar to the virus and good antigenicity and immunogenicity, stimulating generation of endogenous neutralizing antibodies, thus preventing HPV infection (37). The prophylactic vaccine is important in reducing the morbidity and mortality rates of cervical cancer.

In conclusion, the results of this study revealed that OPN had a higher positive expression rate in HPV-positive cervical tumor tissues than in HPV-negative ones, indicating that OPN expression is associated with HPV infection and OPN may be involved in the promoting effects of HPV infection on tumor metastasis. Radical HPV therapy for cervical cancer patients may delay the metastasis of tumor cells and prolong their survival.

\section{References}

1. Luhn P and Wentzensen N: HPV-based tests for cervical cancer screening and management of cervical disease. Curr Obstet Gynecol Rep 2: 76-85, 2013.

2. Regan JA and Laimins LA: Viral transformation of epithelial cells. Methods Mol Biol 945:449-465, 2013.

3. Deberne M, Levy A, Mondini M, Dessen P, Vivet S, Supiramaniam A, Vozenin MC and Deutsch E: The combination of the antiviral agent cidofovir and anti-EGFR antibody cetuximab exerts an antiproliferative effect on HPV-positive cervical cancer cell lines' in-vitro and in-vivo xenografts. Anticancer Drugs 24: 599-608, 2013.

4. Srivastava S, Shahi UP, Dibya A, Gupta S and Roy JK: Distribution of HPV genotypes and involvement of risk factors in cervical lesions and invasive cervical cancer: a study in an Indian population. Int J Mol Cell Med 3: 61-73, 2014.

5. Huh W, Williams E, Bramley T, Huang J and Poulios N: Costeffectiveness of various combinations of human papillomavirus (hpv)-based testing, including genotyping for hpv 16/18, for cervical cancer screening. Value Health 16: A142-A143, 2013.

6. Racey CS, Withrow DR and Gesink D: Self-collected HPV testing improves participation in cervical cancer screening: a systematic review and meta-analysis. Can J Public Health 104: 159-166, 2013.

7. Sadraeian M, Rasoul-Amini S, Mansoorkhani MJ, Mohkam M, Ghoshoon MB and Ghasemi Y: Induction of antitumor immunity against cervical cancer by protein HPV-16 E7 in fusion with ricin $\mathrm{B}$ chain in tumor-bearing mice. Int J Gynecol Cancer 23: 809-814, 2013.

8. Katki HA, Schiffman M, Castle PE, Fetterman B, Poitras NE, Lorey T, Cheung LC, Raine-Bennett T, Gage JC and Kinney WK: Five-year risks of CIN 3+ and cervical cancer among women who test Pap-negative but are HPV-positive. J Low Genit Tract Dis 17 (5 Suppl 1): s56-s63, 2013.

9. Xu Q, Yuan B, Xue F, et al: OPN gene polymorphisms are associated with susceptibility and clinicopatholigical characteristics of cervical cancer in a Chinese cohort. Cancer Biomark 10: 233-239, 2011.

10. Smola-Hess S, Pahne J, Mauch C, Zigrino P, Smola H and Pfister HJ: Expression of membrane type 1 matrix metalloproteinase in papillomavirus-positive cells: role of the human papillomavirus (HPV) 16 and HPV8 E7 gene products. J Gen Virol 86: 1291-1296, 2005. 
11. Herfs M, Parra-Herran C, Howitt BE, Laury AR, Nucci MR, Feldman S, et al: Cervical squamocolumnar junction-specific markers define distinct, clinically relevant subsets of low-grade squamous intraepithelial lesions. Am J Surg Pathol 37: 1311-1318, 2013.

12. Wang S, Pang T, Gao M, Kang H, Ding W, Sun X, Zhao Y, Zhu W, Tang X, Yao Y and Hu X: HPV E6 induces eIF4E transcription to promote the proliferation and migration of cervical cancer. FEBS Lett 587: 690-697, 2013.

13. Branković I, Verdonk P and Klinge I: Applying a gender lens on human papillomavirus infection: cervical cancer screening, HPV DNA testing, and HPV vaccination. Int J Equity Health 12: 14 2013.

14. Chang L, He X, Yu G and Wu Y: Effectiveness of HPV 16 viral load and the E2/E6 ratio for the prediction of cervical cancer risk among Chinese women. J Med Virol 85: 646-654, 2013.

15. Chu A, Genden E, Posner M, and Sikora A: A patient-centered approach to counseling patients with head and neck cancer undergoing human papillomavirus testing: a clinician's guide. Oncologist 18: 180-189, 2013.

16. Priebe AM: 2012 cervical cancer screening guidelines and the future role of HPV testing. Clin Obstet Gynecol 56: 44-50, 2013.

17. Wentzensen N: Triage of HPV-positive women in cervical cancer screening. Lancet Oncol 14: 107-109, 2013.

18. Eide ML and Debaque H: HPV detection methods and genotyping techniques in screening for cervical cancer. Ann Pathol 32: e15-e23, 2012.

19. Ragin C, Obikoya-Malomo M, Kim S, Chen Z, Flores-Obando R, Gibbs D, et al: HPV-associated lung cancers: an international pooled analysis. Carcinogenesis 35:1267-1275, 2014

20. Xu Q, Xue F, Yuan B, Zhang L, Li J and He Z: The interaction between RAGE gene polymorphisms and HPV infection in determining the susceptibility of cervical cancer in a Chinese population. Cancer Biomark 11: 147-153, 2012.

21. Cox JT, Castle PE, Behrens CM, Sharma A, Wright TC Jr and Cuzick J; Athena HPV Study Group: Comparison of cervical cancer screening strategies incorporating different combinations of cytology, HPV testing, and genotyping for HPV 16/18: results from the ATHENA HPV study. Am J Obstet Gynecol 208: 184.e1-184 e11, 2013

22. Sasagawa T, Takagi $\mathrm{H}$ and Makinoda $\mathrm{S}$ : Immune responses against human papillomavirus (HPV) infection and evasion of host defense in cervical cancer. J Infect Chemother 18: 807-815, 2012.

23. Pilch H, Günzel S, Schäffer U, Tanner B, Brockerhoff $P$, Maeurer M, et al. Human papillomavirus (HPV) DNA in primary cervical cancer and in cancer free pelvic lymph nodes - correlation with clinico-pathological parameters and prognostic significance. Zentralbl Gynakol 123:91-101, 2001.
24. Habbous S, Pang V, Eng L, Xu W, Kurtz G, Liu FF, Mackay H, Amir E and Liu G: p53 Arg72Pro polymorphism, HPV status and initiation, progression, and development of cervical cancer: a systematic review and meta-analysis. Clin Cancer Res 18: 6407-6415, 2012.

25. Wang N, Yin D, Zhang S, Wei H, Wang S, Zhang Y, Lu Y, Dai S, Li W, Zhang Q and Zhang Y: TNF-alpha rs1800629 polymorphism is not associated with HPV infection or cervical cancer in the Chinese population. PLoS One 7: e45246, 2012.

26. Wiwanitkit V: Prevalence of HPV genotypes in cervical cancer in Thailand. Int J Gynecol Cancer 22: 1290, 2012.

27. Markman M: Risk of cervical cancer after HPV vaccination. Curr Pharm Des 19: 1488-1489, 2013.

28. Fröberg M, Norman I, Johansson B, Hjerpe A, Weiderpass E and Andersson S: Liquid-based cytology with HPV triage of low-grade cytological abnormalities versus conventional cytology in cervical cancer screening. Curr Pharm Des 19: $1406-1411,2013$

29. Sharma M, Bruni L, Diaz M, Castellsagué X, de Sanjosé $S$, Bosch FX and Kim JJ: Using HPV prevalence to predict cervical cancer incidence. Int J Cancer 132: 1895-1900, 2013.

30. Szarewski A: HPV vaccination and cervical cancer. Curr Oncol Rep 14: 559-567, 2012

31. Keller MJ, Burk RD, Xie X, Anastos K, Massad LS, Minkoff H, Xue X, D'Souza G, Watts DH, Levine AM, et al: Risk of cervical precancer and cancer among HIV-infected women with normal cervical cytology and no evidence of oncogenic HPV infection. JAMA 308: 362-369, 2012.

32. Huang CY, Chen JJ, Shen KY, Chang LS, Yeh YC, Chen IH, Chong P, Liu SJ and Leng CH: Recombinant lipidated HPV E7 induces a Th-1-biased immune response and protective immunity against cervical cancer in a mouse model. PLoS One 7: e40970 2012.

33. Origoni M, Cristoforoni P, Costa S, Mariani L, Scirpa P,Lorincz A and Sideri M: HPV-DNA testing for cervical cancer precursors: from evidence to clinical practice. Ecancermedicalscience 6: 258,2012

34. Schmitz M, Driesch C, Jansen L, Runnebaum IB and Dürst M: Non-random integration of the HPV genome in cervical cancer. PLoS One 7: e39632, 2012.

35. Kaarthigeyan K: Cervical cancer in India and HPV vaccination. Indian J Med Paediatr Oncol 33: 7-12, 2012.

36. Barbisan G, Pérez LO, Contreras A and Golijow CD: TNF- $\alpha$ and IL-10 promoter polymorphisms, HPV infection, and cervical cancer risk. Tumour Biol 33: 1549-1556, 2012.

37. Bosch FX, de Sanjosé S and Castellsagué X: The prospects of HPV vaccination in cervical cancer prevention: results of a new independent trial. Cancer Discov 1: 377-380, 2011. 